\title{
Virtual Community Computer-Telephony
}

\author{
Günter-Ulrich Tolkiehn
}

\section{Aufgabenstellung und Voraussetzungen}

Es war beabsichtigt, mithilfe des Internets und geeigneter Systeme und Anwendungen für Kommunikation und Content-Management kommerziellen Anwendern und Anbietern von Computer-Telefonie-Technik eine themenbezogene interaktive Plattform fuir Information, Kommunikation und Meinungsbildung zu bieten, wobei es öffentliche und Mitgliederbereiche geben soll. Solche Gebilde, virtuelle Interessengemeinschaften, werden auch als vertikales Business-to-Business (B2B)-Portal oder B2B-Virtual Community of Interest bezeichnet. Das Themengebiet „Computer-Telephony“ ist vom Ansatz her auf Firmen und Personen ausgerichtet, die als Hersteller, Dienstleister oder Nutzer von Technik bzw. Software in diesem Bereich auftreten (B2B-Community). Damit ist auch die Zielgruppe definitiv eingeschränkt.

Das Vorhaben geht zurüick auf eine gemeinsame Idee von Dr. Volker Puke (Tieto-Enator Consulting) und dem Projektleiter aus dem November 1997, ein anbieterunabhängiges Informations- und Diskussionsforum im Internet für den Themenbereich Computer-Telefonie zu schaffen. Nach anfänglicher Unschlüssigkeit, wie das Vorhaben technisch und organisatorisch zu realisieren sein könnte, wurden dann in 1998 die Entscheidungen fuir die Vorbereitung einer Realisierung im Rahmen des Instituts für Telekommunikation an der Technischen Fachhochschule Wildau (TFH Wildau), fuir die Form einer Virtual Community und für die Verwendung eines kommerziellen Content-Management-Systems getroffen.

Anfang 1998 war bereits vorsorglich die Domain www.computer-telephony.de reserviert worden. Um direkte Überschneidungen mit einer eventuellen Internationalisierung der Homepage der damaligen US-Zeitschrift Computer Telephony Magazine (www.computertelephony.com) zu vermeiden, zu der es dann nach dem Verkauf des Computer Telephony Magazines an Miller-Freeman durch den deutschen Telepublic Verlag Ende 1998 auch tatsächlich kam, wurde von vornherein die Schreibweise mit Bindestrich gewählt.

Ende 1998 wurde nach einigen Vorüberlegungen ein Antrag der TFH Wildau auf Förderung durch das Bundesministerium fuir Bildung und Forschung (BMBF) im Rahmen des Programms der Arbeitsgemeinschaft Industrieller Forschungsvereinigungen „Otto von Guericke“ e. V. (AiF) gestellt, der im Sommer 1999 genehmigt wurde. Im 1. Quartal 1999 stellte die Hyperwave AG dem Institut im Hinblick auf das beantragte Vorhaben bereits eine für drei Jahre unentgeltliche Systemlizenz für das zu der
Zeit führende objektorientierte Web-Content-Management-System Hyperwave Information Server (HIS) zur Verfuigung, das an der Uni Graz entwickelt worden war.

Ein erstes Serversystem (PII 233) mit Windows NT 4.0 für Testzwecke und Prototyping wurde dem Institut fuir vorbereitende Tests von der ebenfalls an dem Projekt interessierten Karlsruher PRS-GmbH (inzwischen leider in Zusammenhang mit dem Metallbank-Zusammenbruch nicht mehr im Geschäft) gespendet.

Ein etwas leistungsfähigeres, jedoch immer noch bescheidenes zweites NT-System für das Vorhaben beschaffte im Mai 1999 das Institut für Telekommunikation an der TFH Wildau aus eigenen Mitteln. Der erste Server wird seitdem für zusätzliche Anwendungen verwendet, die z. T. auch allgemeinen Aufgaben der Lehre an der TFH Wildau dienen. Bereits im Sommersemester 1999 wurden mit einer Gruppe von Studenten experimentell eine vorläufige Struktur erarbeitet und Tests mit ersten Inhalten auf dem HIS durchgefuihrt.

Bei Projektstart lagen uns weder Erfahrungsberichte noch verwertbare Veröffentlichungen oder andere Kenntnisse über deutschsprachige B2B-Communities vor. Die zu der Zeit bekannten Beispiele von Virtual Communities basierten im Wesentlichen auf privaten Interessen (Hobbys, Selbsthilfegruppen für Krankheiten und Behinderungen etc.) und waren im englischen Sprachraum (i. W. USA) angesiedelt. Wir waren daher darauf angewiesen, eigene Überlegungen über Nutzen, Inhalte, Technik und Handhabung anzustellen.

\section{Planung und Ablauf des Vorhabens}

Das Vorhaben wurde inhaltlich und technisch voll im Rahmen der Planung umgesetzt. Aufgrund der schwierigen Personalsituation (siehe dazu unten unter „Personal“) kam es zu einem um zwei Monate verzögerten Start (was den Eintritt des Vollzeit-Projektmitarbeiters betrifft) und in Abstimmung mit dem Projektträger zu einer Verschiebung des Projektendes um drei Monate auf den 31.05.2001.

\subsection{Konzeption}

Aufgrund erheblicher Vorarbeiten lagen bei Projektbeginn bereits viele Vorüberlegungen und Elemente für die Konzeption des Systems vor. Dadurch konnte die Virtual Community www.computer-telephony.de bereits im Oktober 1999 im Sinne von „rapid prototyping“ erst- 
malig - mit noch eingeschränktem Funktionsumfang, nur geringem Informationsinhalt und auf nur eingeschränkt leistungsfähiger Technik - in das Web gestellt werden. Es galt nun, das System parallel konzeptionell und funktional zu entwickeln, mit relevanten Inhalten zu füllen und den potenziellen Nutzern und möglichen Sponsoren bekannt zu machen.

Im Jahr 2000 wurden die wesentlichen Konzepte überprüft und teilweise überarbeitet. Das Konzept des Zugangs zum System wurde nach dem ersten Feedback von Benutzern Anfang 2000 dahingehend verändert, dass wesentlich mehr Informationsbereiche für anonyme Benutzer geöffnet wurden und die Funktionen für angemeldete Benutzer mehr auf die aktive Einbringung von Inhalten und einige Sonderfunktionen und -bereiche konzentriert wurden.

Ebenso stellte sich heraus, dass die Durchsuchbarkeit des Systems durch Internet-Suchmaschinen verbessert werden musste, und zwar sowohl in Hinsicht auf den Zugang der Robots zu den Inhalten zur Aufnahme in die Indizes als auch auf die Angabe des Ziel-URLs, was zunächst wegen der Verwendung der Frame-Technologie immer nur auf die Startseite zeigte. Gleichzeitig zeigte sich im Laufe des Betriebs, dass einige der vorgesehenen Funktionen uninteressant und einige andere schwer zu realisieren bzw. aufrechtzuerhalten waren, wieder andere zeigten kleinere oder auch schwerwiegende Mängel. Daneben wurde das allzu spartanische und teilweise recht uneinheitliche optische Erscheinungsbild kritisiert.

Vom 4. Quartal 2000 bis zum Ende des Förderzeitraums wurde dann das Design der Benutzeroberfläche des Systems unter Berüicksichtigung der neuen Erkenntnisse neu spezifiziert und grundlegend erneuert. Sie wurde sowohl grafisch als auch funktional in die im wesentlichen noch heute laufende Form gebracht.

Wesentliche funktionale Verbesserungen in diesem Zeitraum waren die Neustrukturierung und Neugruppierung aller Menuis (als Pull-Down-Menuis in JavaScript) und Funktionen unter Einfuihrung eines professionellen grafischen und Web-Designs und Verzicht auf Frame-Technologie (auch um in jedem Bereich Bookmarking zu ermöglichen), der Einbau einer Navigationsleiste und die Erstellung von an die jeweiligen Inhalte angepassten Eingabe- und Pflegeformularen mit hinterliegenden Templates.

\subsection{Technik}

Nach mehreren Tests und Vergleichen im Vorfeld des Projekts hatte sich die Arbeitsgruppe Computer-Telefonie zu Projektbeginn bereits fuir den Hyperwave Information Server (HIS) der Hyperwave AG, München, als technische Grundlage für das Portal entschieden. Dieser Webserver basiert vollständig auf Internet-Standardtechnologien und ist als Lösung für komplexes Dokumenten- und Wissensmanagement im Intra- und Extranet konzipiert. Das System (damaliger Lizenzpreis
45.000 DM) wurde vom Hersteller für die Projektlaufzeit unentgeltlich gegen Abschluss eines Supportvertrages und Schaltung eines Links zum Hersteller zur Verfuigung gestellt. Nach anfänglichen technischen und Supportschwierigkeiten und der Einspielung des neuen Releases 5.1 im Dezember 2000 wird das System nunmehr gut beherrscht und läuft recht stabil. Für bestimmte Bereiche der Software-Entwicklung wird jedoch aus praktischen und Sicherheitsgründen ein zweiter, weniger leistungsfähiger Test-Server benutzt, der aus lizenzrechtlichen Gründen nicht über Internet zugänglich ist.

Im Jahr 2000 zeigte sich dann in Zusammenhang mit den wechselnden Mitarbeitern auch bereits eine Schwäche der zu Grunde liegenden Systemtechnologie. Es erwies sich als schwierig, die zur Gestaltung der Benutzeroberfläche und der Funktionselemente erforderlichen Templates und Scripte mithilfe der Systemdokumentation und des Supports so zu erstellen, dass eine stabile Funktion gewährleistet werden konnte, und ebenso, sie so zu dokumentieren, dass ein anderer Entwickler sich ohne weiteres in die bereits bestehenden Elemente einarbeiten konnte.

Dies fuihrte zu einem mit Sicherheit suboptimalen Ergebnis der Programmierung. Im Ergebnis liefert das bestehende System einerseits relativ schlechte und ungleichmäßige Antwortzeiten, die weitgehend unabhängig von der übertragenen Datenmenge und -rate für die PageImpression waren. Sie liegen im Bereich von 12 bis 40 Sekunden, hierzu wurden ausfuihrliche Messungen gemacht, siehe dazu exemplarisch Abbildung 1, in dem Messungen über einen ISDN-Zugang mit $64 \mathrm{kbit} / \mathrm{s}$ über T-Online mit verschiedenen Seiten, Einstellungen, Browsern und mit oder ohne Verwendung eines lokalen Proxy-Servers sowie auch unter Ausschaltung des Browser-Cache gemacht wurden. Dieses Problem konnte erst nach Projektende durch die akribische Feinarbeit des Jaroslawler Gastwissenschaftlers Tykin gelöst, wenn auch nicht im Detail verstanden werden. Seit Mai 2002 liegen die Antwortzeiten des Systems nun bei den für solche Datenbankserver weltweit üblichen drei bis zehn Sekunden.

Andererseits stürzte das System bereits mehrfach (ca. einmal im Halbjahr) aufgrund von bisher ungeklärten internen Problemen des HIS komplett ab, was im letzten Fall (Januar 2002) sogar zu Datenverlust fuihrte.

Ebenso zeigte sich bei Prüfung mittels Link-CheckerSoftware, dass die Link-Konsistenz des Systems, die eigentlich vom HIS automatisch sichergestellt werden müsste, häufiger erhebliche Lücken aufwies (sowohl Objekte, die nicht mehr uiber Links angesprochen werden konnten, oder Links, zu denen keine Objekte mehr vorhanden waren). Eine Ende 2000 durchgefuihrte Untersuchung der technischen Alternativen, insbesondere von Microsoft ASP, führte aber zu dem Ergebnis, dass ein Umstieg einen im Rahmen des Projektplans unvertretbar hohen Aufwand erfordert hätte. An der Verbesserung von Performance und Stabilität der Datenbank wird mit Unterstuitzung des Hyperwave Supports derzeit noch 


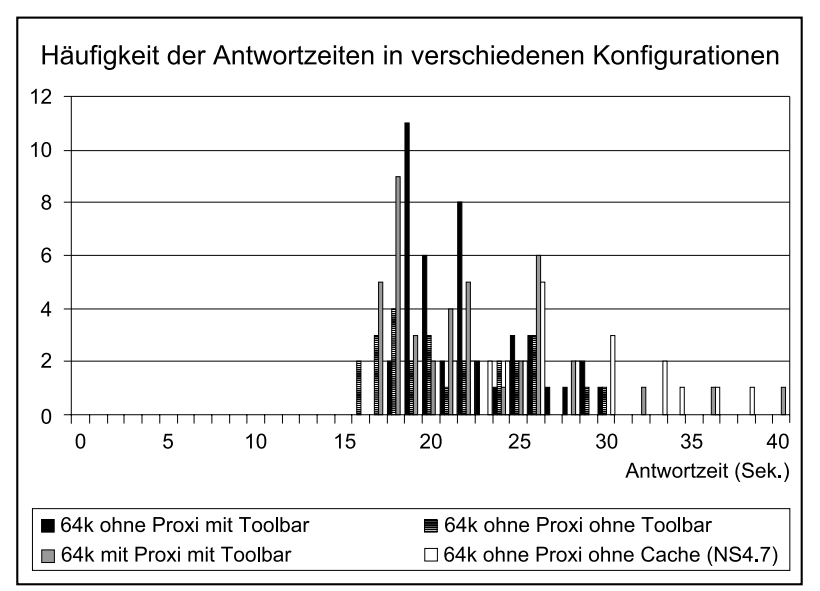

Abb. 1: Häufigkeitsverteilung der Antwortzeiten

gearbeitet. Auch der Anteil von 0,7 \% fehlerhaften Zugriffen aufgrund von Serverfehlern (davon 0,5\% Gateway-Timeout und 0,2\% interne Serverfehler (Error 500) im Mittel über das Jahr 2001) lässt noch auf gewisse softwaretechnische Schwächen im Server schließen.

Während die Hardware des Produktionssystems von vornherein und nach der Umstellung von Windows NT auf Windows 2000 auch das Betriebssystem erfreulich stabil liefen, blieben wir trotz Verwendung aktueller Virenscanner auch nicht von den für alle Benutzer von Servern mit Microsoft-Technologie schwierigen Angriffen durch die Würmer „code red“ und später „Nimda“ verschont. Zusätzlich gab es eine Reihe von Abschaltungen, die auf TFH-internen Problemen beruhten (Stromausfälle, Arbeiten am Netzwerk, Ausfälle des Internetzugangs, Abschaltungen wegen Bauarbeiten auf dem Gelände), sodass wir insgesamt eine Verfügbarkeit des Systems von ca. 95 \% nicht überschreiten konnten, was in diesem Bereich noch als akzeptabel gelten kann.

Die Sicherung der gesamten Daten erfolgt tageweise dreischichtig bedienerlos auf Platte uiber das LAN. Eine weitere Sicherung erfolgt in größeren Abständen auf dem zweiten, nur lokal zugreifbaren Server (das Entwicklungs- und Testsystem). Seit Januar 2002 wird nach Anschaffung eines entsprechenden Geräts aus Mitteln der TFH zusätzlich monatlich auf einen DAT-Streamer gesichert.

\subsection{Public Relations und Gewinnung von Benutzern}

Aus Erfahrungen kommerzieller Websites und aus der Literatur war bekannt, dass insbesondere in der Anfangsphase nur relativ geringe Besucher- und Page-Impression-Zahlen und nur sehr geringe Mitgliederzahlen erwartet werden durften und dass auch unter optimalen Randbedingungen eine relativ lange Zeit (ca. fünf Jahre) bis zu einer eigendynamischen Entwicklung des Systems zu erwarten ist. Deshalb werden neben dem Aufbau der initialen Inhalte und der Pflege einiger aktueller Rubriken die für diese fachspezifisch orientierte Community wichtige Interessengruppen gezielt und kontinuierlich angesprochen. Eine Ansprache uiber Werbung ist ohne nennenswertes Werbebudget vor allem über die weni- gen deutschen Fachzeitschriften für Computer-Telefonie, Call Center und VoIP sowie uiber die Präsentation auf speziellen Events möglich.

Parallel zur Inbetriebnahme des ersten Prototyps am Netz wurde daher mit der Bewerbung der Community auf Fachveranstaltungen und Messen der Computer- und Internetbranche sowie im Bereich der persönlichen Kontakte des Projektteams begonnen. Die Community wurde auf den IT-Messen Systems 99, Exponet 99, CeBIT 2000, Systems 2000, Exponet 2000 und CeBIT 2001 präsentiert, teilweise im Rahmen der Präsentation der TFH Wildau auf den Gemeinschaftsständen des Landes Brandenburg, teilweise auf besonderen Ständen sowie in einer Reihe von Konferenzbeiträgen und im Telecomunications Newsletter der Münchner dc Congress Gesellschaft der Fachöffentlichkeit vorgestellt. Eine intensive Bewerbung dieser Messeauftritte im Vorfeld über die uiblichen Organe der Messeveranstalter hinaus war aufgrund des Budgets natuirlich nicht möglich. Dennoch war der Zuspruch an Interessenten zufriedenstellend.

In erster Linie war mit diesen Aktivitäten beabsichtigt, Firmenmitarbeiter als Multiplikatoren zu gewinnen, um hier bereits in der Anfangsphase insbesondere Anbieter von Produkten und Dienstleistungen als aktive Nutzer und Mitgestalter der Internet-Site zu gewinnen. Dies gelang auch in erheblichem Umfang. Ende März 2000 zählte die Community bereits ca. 150 registrierte User. Bremsende Faktoren dabei waren einerseits die noch bis Ende März erheblichen programmtechnischen Probleme, die noch unvollständigen Inhalte sowie häufig nur passives Interesse oder eine gewisse Schwellenangst vor einer aktiven Mitwirkung bei den Usern. Dennoch wurden bereits nach dieser kurzen Zeit erste Inhalte von Dritten eingestellt. Die Zahl der registrierten Benutzer stieg dann kontinuierlich und erreichte zum Ende des Förderzeitraums den Wert von 350 und überschritt die 450 im März 2002.

\subsection{Personal}

Günstig wirkte sich aus, dass wir bereits zu Beginn des Projekts einen Doktoranden der Partneruniversität Jaroslavl (Russland) zur Verfuigung hatten, der theoretische Aspekte des Vorhabens zum Themas seiner Dissertation machte, aber auch praktische Programmierarbeiten in erheblichem Umfang leistete, ohne das Projektbudget zu belasten. Weiterhin war es sehr vorteilhaft, dass sowohl die technische Infrastruktur (LAN, Internet-Zugang, Laborräume) bereits vor Beginn des Projekts zur Verfuigung standen und dass eine sehr qualifizierte Kraft für die Systemadministration und die technische Spezifikation und Auswahl der zu beschaffenden Komponenten von Seiten der TFH Wildau während der gesamten Projektlaufzeit zur Verfuigung stand.

Es erwies sich jedoch - auch aufgrund der damals sehr angespannten Marktsituation fuir Internet-Fachleute - als schwierig, den für das Projekt vorgesehenen wissenschaftlichen Mitarbeiter/in zu gewinnen. Erschwerend wirkte sich ebenfalls aus, dass die Personalsuche mit 
extrem kurzer Vorlaufzeit erfolgen musste, da der Zuwendungsbescheid für das Projekt erst zu Beginn der Sommerferien erging und Projektstart der 01.09. sein sollte.

Die Beschäftigung von studentischen Hilfskräften war zu den vorgesehenen Stundensätzen ebenfalls schwierig. Wir umgingen dieses Problem zum Teil dadurch, dass wir geeignete Teile der eigentlich für studentische Hilfskräfte vorgesehenen Arbeiten in unser Projektstudium Wirtschaftsinformatik einbrachten, was sehr guten Anklang bei den Studenten fand, sodass ein Teil dieser Arbeiten erbracht werden konnte, ohne das Projektbudget zu belasten. Es gelang schließlich per 27.10.1999, eine überdurchschnittliche Absolventin der TFH für die Projektleitung zu gewinnen. Sie verließ das Projekt jedoch - verständlicherweise - als sie zum 01.04.2000 eine finanziell wesentlich attraktivere Dauerstelle in einem Unternehmen angeboten bekam.

Glücklicherweise konnten wir nahtlos einen wissenschaftlichen Mitarbeiter für das Projekt gewinnen, der bereits auf einer Zeitstelle in der TFH arbeitete, inhaltlich eingearbeitet war und bereits in der Planungsphase und begleitend in kleinerem Umfang an dem Projekt mitgewirkt hatte. Leider machte in diesem Fall die Hochschulverwaltung erhebliche Schwierigkeiten, dem Mitarbeiter die vorgesehene Einstufung nach BAT IIa Ost zu gewähren, obwohl er alle Voraussetzungen erfuillte, und versuchte schließlich, ihm im Gegenzug projektfremde Verpflichtungen aufzuerlegen, was nicht motivierend wirkte. Auch dieser Mitarbeiter verließ uns vorzeitig zum 30.09.2000, als er eine Dauerstelle an der Universität Potsdam angeboten bekam. Er arbeitet jedoch bis heute - unbezahlt und natürlich nur in kleinem Umfang - weiter an dem Projekt mit. Leider bekam etwa gleichzeitig der angehende russische Doktorand ein Green-Card-Angebot der Volkswagen AG in Wolfsburg im Bereich Content-Management und WebDesign, das er unmöglich ablehnen konnte, und entschwand ebenfalls unter Abbruch seiner Doktorarbeit zum 30.9.2000, was eine kritische Situation für das Projekt zur Folge hatte.

Nur durch die aktive Unterstuitzung unserer Partneruniversität in Jaroslavl gelang es dann, kurzfristig zwei Assistenzprofessoren aus Jaroslavl fuir die restliche Projektlaufzeit abzustellen. Dadurch konnte, wenn auch unter der schwierigen Randbedingung, dass die beiden zunächst ohne jede Kenntnis der deutschen Sprache an einem deutschsprachigen Content-Management-System arbeiten mussten, der erfolgreiche Abschluss der Redesign- und Programmierarbeiten sichergestellt werden. Dass dadurch in erheblichem Umfang andere Projektarbeiten außerplanmäßig auf den Projektleiter und die Systemadministratorin entfielen, musste in Kauf genommen werden. Die beiden russischen Mitarbeiter haben nach Projektende ebenfalls Green-CardAngebote eines deutschen Software-Unternehmens angenommen, stehen aber ebenfalls noch in kleineren Umfang fuir gelegentliche Entwicklungsarbeiten an dem System bereit.

\section{Die wesentlichen wissenschaftlich- technischen Ergebnisse}

Das System ist in der im Folgenden beschriebenen Form zum Ende der Projektlaufzeit (Mai 2001) fertiggestellt worden und läuft seitdem - mit kleineren funktionalen Verbesserungen und bei ständiger Aktualisierung der Inhalte - im Wesentlichen unverändert. Auch die Präsentation der Community in Fach-Vorträgen (zuletzt auf der VoIP-Konferenz des Anwenderkreises Informationstechnik AKIT in Hannover im Februar 2002) wird weiterhin kontinuierlich fortgesetzt.

\subsection{Inhalte und Gliederung des Systems}

Grundsätzlich soll in allen Content-Bereichen die Einbringung der Inhalte durch Mitglieder erfolgen. Dazu müssen entsprechend benutzerfreundliche Eingabe- und Änderungswerkzeuge angeboten werden. Diese Entwicklung ist in vielen, aber noch nicht in allen Bereichen abgeschlossen. In den anderen Bereichen sind bisher nur die etwas anstrengenden Standardeingabefunktionen von Hyperwave verfuigbar. Nur wenige dieser Bereiche (im Wesentlichen Fachartikel, Seminare und Events und News) erfordern eine ständige Pflege durch die Redaktion. Allerdings muss zunächst und über längere Zeit der Aufbau von initialen Inhalten betrieben werden, um die für einen erfolgreichen Betrieb erforderliche kritische Masse an Information und Nutzen zu überschreiten.

Im Jahr 2000 wurden die wesentlichen Inhalte ausgearbeitet sowie die konzeptionellen Weiterentwicklungen umgesetzt. Zum Projektende war dann das grafische und funktionale Layout abgeschlossen. Das gegenwärtige Aussehen der Startseite zeigt die Abbildung 2:

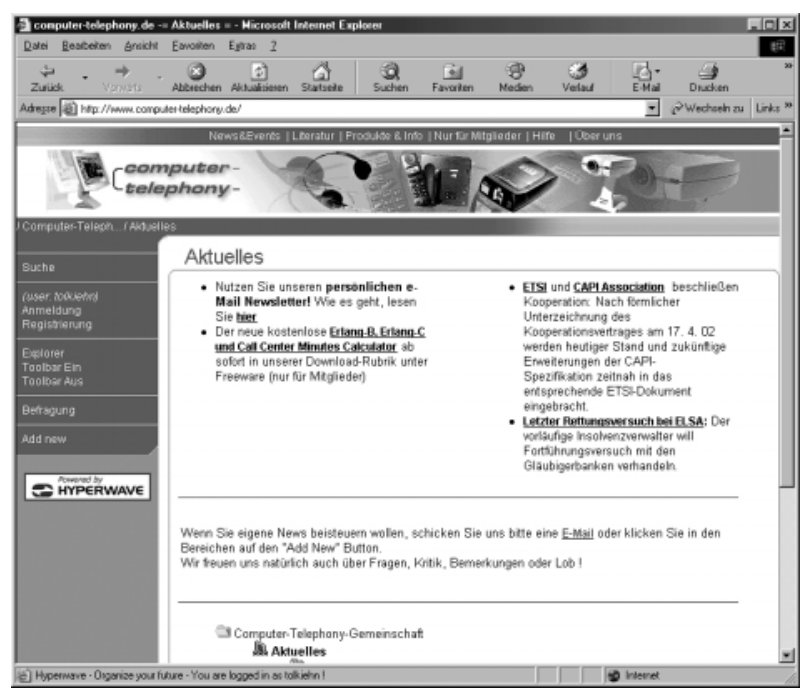

Abb. 2: Screenshot Aktuelles (Startseite)

Zum Ende des Förderzeitraums waren im Einzelnen die unten folgenden Bereiche, Inhalte und Funktionen implementiert. Die Screenshots zeigen den Stand der Inhalte vom März 2002, der Seitenaufbau ist im Wesentlichen identisch mit dem Stand vom Mai 2001. In den Screenshots wird jeweils die Ansicht fuir angemeldete Benutzer gezeigt. 


\section{News und Events mit den Bereichen}

- Aktuelles - die Startseite der Community mit Neuigkeiten aus dem Bereich mit folgenden Themenunterteilungen: Auf der Titelseite Top-News, auf den weiteren Seiten:

- Business \& Börse, Call-Center, CRM,

- CTI Hardware,

- CTI Software,

- IVR, UM, Spracherkennung, Voice-over-IP

- Mobile Kommunikation

- Standards

- Telekommunikation \& Sonstiges

- Veranstaltungstermine

- VideoConferencing \& ApplicationSharing

- Seminare und Events: Chronologisch geordnete Kurzinformationen und darin Links zum Programm (soweit im Netz) zu Seminaren, Kongressen, Messen und sonstige Veranstaltungen zum Thema. Das Aussehen der Übersicht zeigt Abbildung 3:

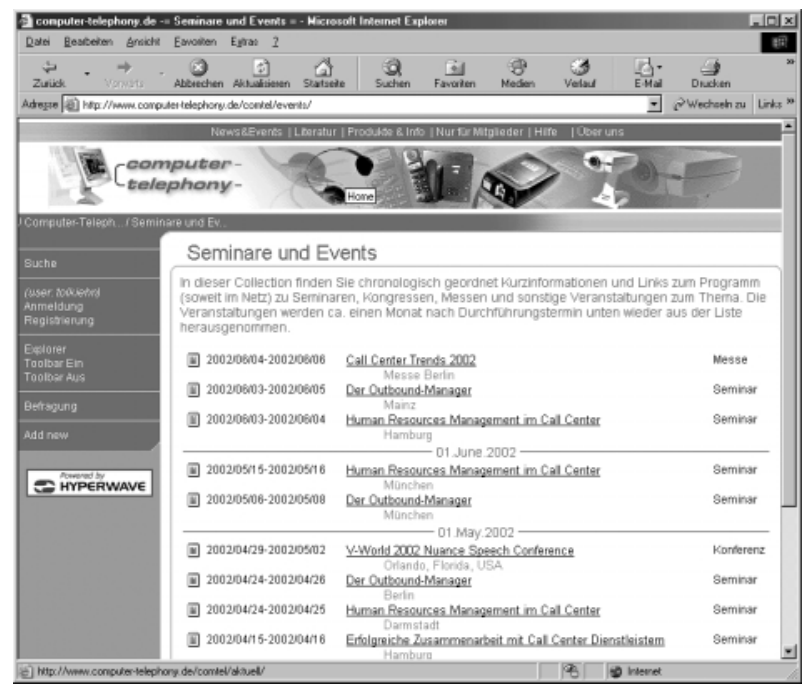

Abb. 3: Screenshot Veranstaltungskalender Übersicht

Hier war es wichtig, die wichtigen Kurzinformationen festzulegen und knapp und übersichtlich im Zusammenhang darzustellen. Die Darstellungsform wurde mehrfach revidiert, bis sich die im Bild angegebene Form entwickelt hatte. Es werden sowohl in der Übersicht als auch in der unten gezeigten Einzelinformation nur ausgewählte Attribute des jeweiligen Objekts zur Darstellung verwendet. Auf die Ablage von Dateien wurde in dieser Collection und ebenso in einigen anderen ganz verzichtet.

Die einzelnen Kurzinformationen haben dann templategesteuert ein einheitliches Aussehen und einheitlichen Aufbau, was in Abbildung 4 beispielhaft gezeigt wird. Alte Veranstaltungen blenden sich per Ablaufdatum (standardmäßig auf sechs Wochen nach dem Veranstaltungsende eingestellt) automatisch aus der Übersicht aus und werden zur weiteren Verwendung (z. B. bei Wiederholungen) und zur späteren Analyse in dem versteckten Ordner ,alte Veranstaltungen“ gesammelt.

Die Eingabemöglichkeit für neue Objekte über den Button ,add new“ erzeugt eine Formularseite, die auf die jeweiligen Inhalte und Attribute des Objekts angepasst

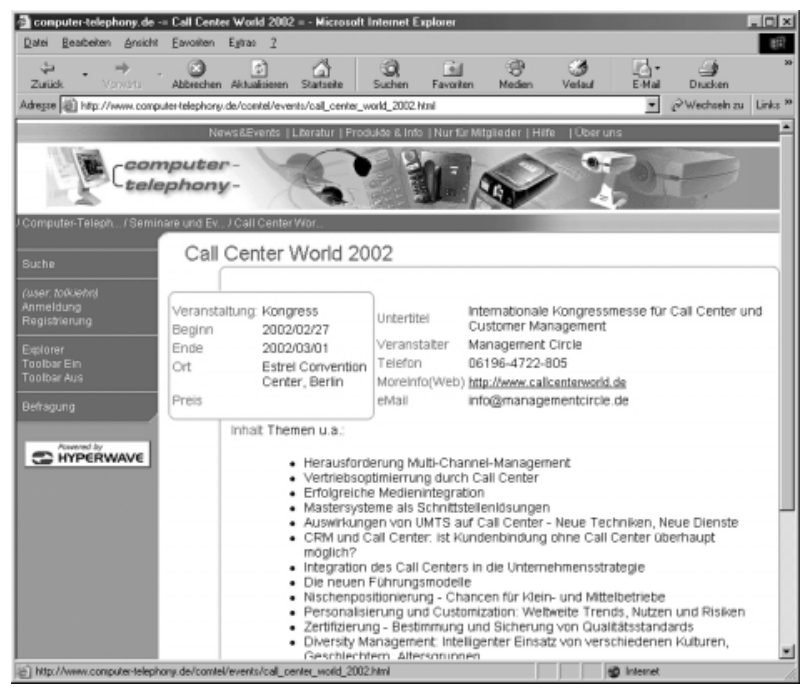

Abb. 4: Screenshot Beispiel einer Veranstaltungsinformation

ist. Beispielhaft wird hier in Abbildung 5 die Seite für die Eingabe einer Veranstaltung in „Seminare und Events“ gezeigt. Die Angaben zum Attribut „Inhalt“ können in der letzten Version des Eingabeformulars bei Verfuigbarkeit einer Webseite des Anbieters zur Veranstaltung direkt auszugsweise per Drag-and-Drop als HTML-Code aus dieser übernommen werden, was die Wahrscheinlichkeit von Tippfehlern bei der Eingabe eliminiert.

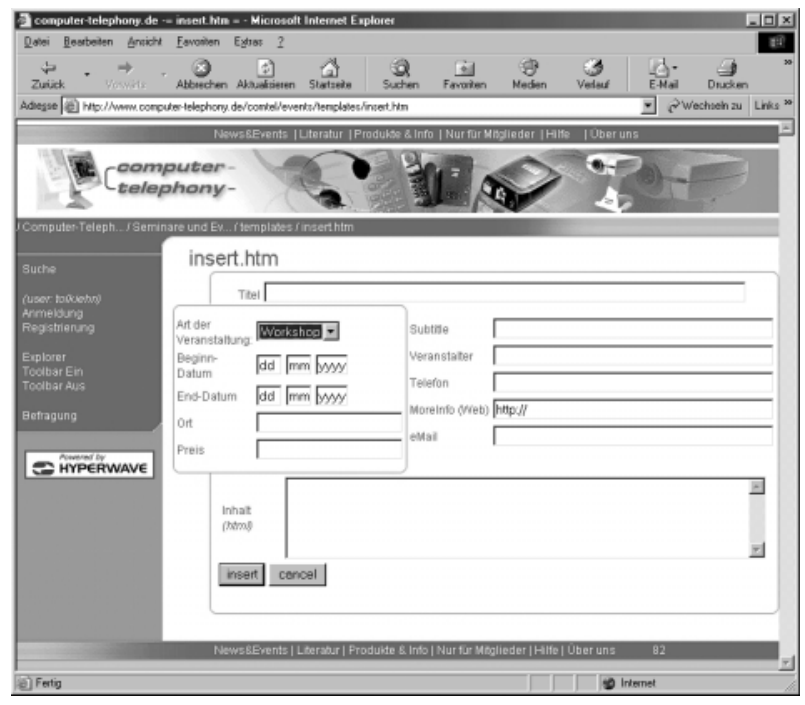

Abb. 5: Screenshot Beispiel Eingabefenster (Seminare und Events)

Die Gestaltung der Eingabeformulare mithilfe der systemeigenen Scriptsprache erwies sich als aufwändig. Die Eingaben erfolgen mit den üblichen HTML-Formular-Funktionen und können auf Wertebereich und Syntax geprüft werden. Alle diese Prüfungen müssen jedoch bei der Gestaltung eines Formulars über Templates von Hand programmiert werden. Die Sicherheit gegen fehlerhafte Eingaben wurde bisher noch nicht in jedem Fall in wirklich zufriedenstellendem Umfang erreicht. Als besonders heikel erwies sich die automatische Generierung des Dateinamens bzw. Pfadelements aus dem Titel, da hierbei systemseitig keine Prüfung auf unzulässige Zeichen vorgenommen wird, so dass standardmäßig ungültige URLs generiert werden, wie zum Beispiel „.../Münchner\% 20Workshop.html“. Das 
gleiche Formular wird auch zur Änderung eines Objekts verwendet.

\section{Literatur mit den Bereichen}

- Literaturübersicht mit den Unterbereichen

- Bücher (dieser Unterbereich konkurriert genauso wie CD-ROM mit den kommerziellen Angeboten von E-Shops wie BOL oder Amazon)

- CD-ROM

- Firmenschriften

- Sonstige Literatur (ohne ISBN-Nummer, z.B. Diplomarbeiten, Dissertationen)

- Standards

- Whitepapers und andere allg. Firmenschriften - Zeitschriften: Versuch, einen Überblick über die themenrelevanten Fachzeitschriften zu geben

- Inhalte Fachartikel: Ein Leseservice mit Kurzfassungen von Fachartikeln aus sieben ausgewählten Fachzeitschriften: Themenrelevante Artikel in einschlägigen Fachzeitschriften wie Call-Center Magazine, Computer Telephony Magazine, Computer Telephony (deutsch), TeleTalk, Voice+ wurden mit Titel, Autor und Kurzangabe zum Inhalt sowie ggf. Links auf den kompletten Artikel im Web zusammengestellt.

Die Pflege dieses Bereichs wurde wegen des hohen Aufwands bei Projektende aus wirtschaftlichen Gründen vorläufig eingestellt. Man benötigt etwa acht bis zehn Minuten Arbeitszeit eines kompetenten Studenten für die Bewertung und Einstellung eines Artikels. Gespräche mit Verlagen uiber die aktive Betreuung dieses Bereichs waren naturgemäß unergiebig. Der Bereich wird vermutlich in Kürze zu Gunsten der Ergänzung der Rubrik Zeitschriften in Literaturhinweise um besondere Hinweise auf die zunehmend vorhandenen Online-Ausgaben ganz eingestellt werden.

\section{Produkte und Info mit den Unterbereichen}

- Marktübersicht mit Hinweisen auf und Auszügen aus einer Vielzahl von Studien und Marktuntersuchungen zum Themenbereich, so weit sinnvoll gruppiert nach den einschlägigen Marktforschungsunternehmen

- Reports mit Berichten über Trends und Konzepte

- Produktdatenbank mit Produkten von bisher ca. 38 Anbietern. Abbildung 6 zeigt das Aussehen der Übersicht über die enthaltenen Firmen.

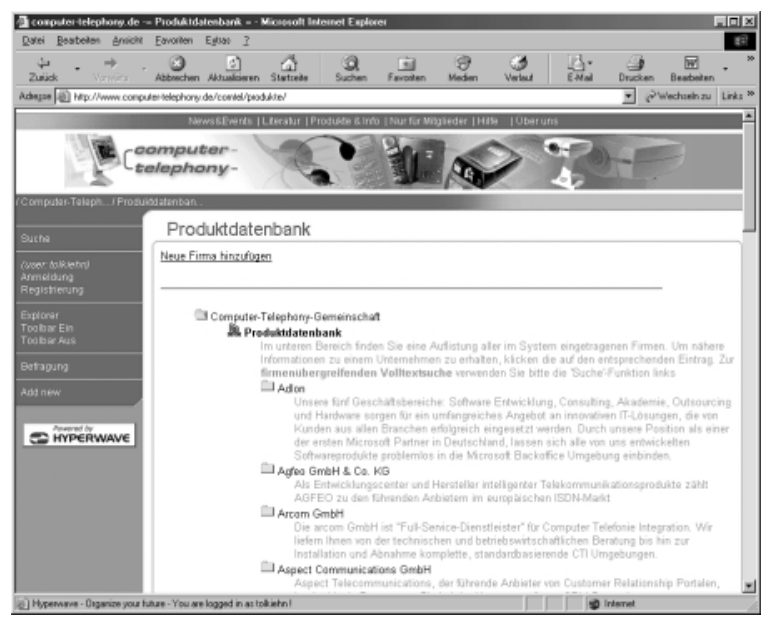

Abb. 6: Screenshot Produktdatenbank Firmenübersicht
Die einzelnen Firmen können ihre Produkte dort einpflegen und mit Kurzbeschreibungen und Links auf die Produkthomepage versehen. Gestaltung und Attribute einer solche Kurzinformation über ein Produkt zeigt Abbildung 7:

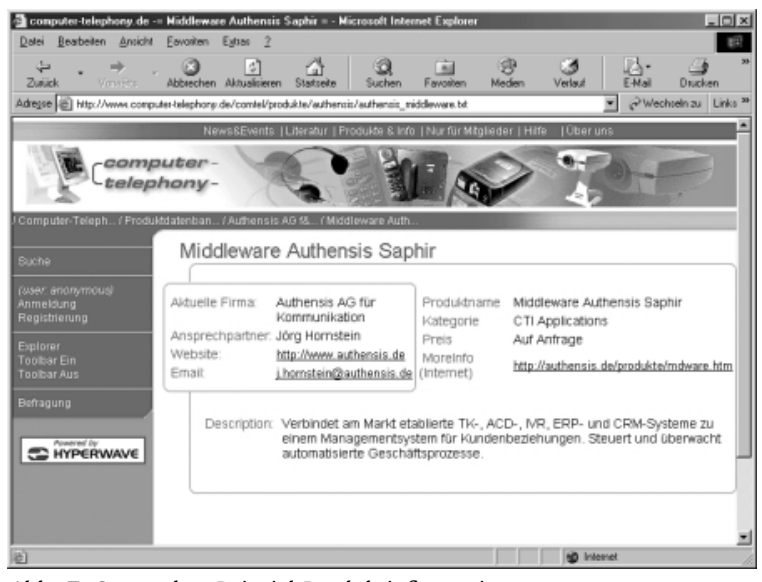

Abb. 7: Screenshot Beispiel Produktinformation

Die Kurzinformationen dienen neben der Information über das Produkt auch dazu, eine einfache Auffindbarkeit des Produkts im System per Volltextsuche zu ermöglichen. Nur der registrierte und von der Projektleitung überpruifte Eigentuimer des Firmenordners erhält die Möglichkeit, Produkte hinzuzufuigen, zu löschen und zu editieren.

- CT-Seiten im Web - mit kommentierten Links auf andere themenverwandten Seiten im Web gruppiert nach den Bereichen:

- Call Center und CRM Sites

- Computer Telephony Sites

- ISDN Sites

- Spracherkennungs-Sites

- Telekommunikations-Sites

- VolP Sites

\section{Nur für Mitglieder mit den Bereichen}

- Forum: Hier können Mitglieder zu freien Themen Diskussionen anlegen und führen. Es handelt sich um eine Funktion des HIS, die leider noch nicht ganz den heute im Web üblichen Standards entspricht.

- Chat: Zur unmoderierten Kommunikation der Mitglieder untereinander. Hierfür wurde ein an der TFH entwickelter Chatserver auf Java-Basis eingesetzt.

- Jobs: Hierfuir wurde zunächst eine Standardfunktion des HIS eingesetzt, die Pinwand, die sich jedoch als unzweckmäßig erwies. Auch für diesen Bereich wurde deshalb eine eigene, designkonforme Eingabeund Darstellungsfunktion erstellt. Jedes Mitglied kann Jobangebote oder -gesuche einstellen. Der Bereich wurde jedoch nie in größerem Umfang von den Mitgliedern benutzt.

- Verkauf: Dieser Bereich wurde zunächst mit einem einfachen Shop hinterlegt, in dem beabsichtigt war, von Studenten erstellte kleinere Softwarekomponenten, wie z. B. Java-Applets, zu verkaufen. Dies erwies sich jedoch gegen Projektende als nicht systemkonform und zu schwierig in der Handhabung und wurde daher eingestellt. De Punkt wird in Kürze aus dem Menui entfernt. 
- Downloads: Ein Bereich mit Daten und Software: Freeware, Hyperwave Tools, Internet-Phone, VoIPProdukte und Dienste, Demos, Präsentationen zum Download, Shareware, Updates und Publikationen, die den Mitgliedern in Abstimmung mit den Inhabern der Copyrights zur Verfuigung gestellt werden.

\section{Hilfe mit den Bereichen}

- Einführung - mit Erklärungen und Infos zu Konzepten, Standards und Hintergründen

- FAQ, häufig gestellte Fragen zum Themenbereich CT und zur Bedienung der Website selbst

- Glossar - Eine Sammlung von Standardkürzeln, Abkürzungen und Begriffen aus dem Themenumfeld

- Hilfe mit praktischen Angaben zur Benutzung der Website, die systembedingt in vielen Bereichen leider nicht völlig selbsterklärend ist

\section{Über uns}

mit den üblichen Angaben zu den Verantwortlichkeiten und Ansprechpartnern des Systems, Haftungsausschluss, Impressum, Mission Statement, unsere Sponsoren

\subsection{Funktionen des Systems}

Die in der Folge aufgefuhrten Funktionen wurden kontextabhängig in der linken Spalte des Fensters als Link zur Verfügung gestellt, sodass die Anmutung einer Frameset-Seite erzielt wird. Die Funktionen sind wie das Seitendesign vom System vorgegeben und können von den Benutzern nicht verändert werden.

- Suche: Der HIS enthält eine leistungsfähige Suchmaschine, die dem Benutzer jederzeit zur Verfuigung steht. Die Suchmaschine durchsucht entweder den gesamten Server oder den aktuellen Scope und ist in der Lage, sowohl Attribute der vorhandenen Objekte (z. B. das Datum der Erstellung) als auch Volltextsuche in sämtlichen gängigen Dateiarten (z. B. auch Powerpoint-, pdf- oder Word-Dateien) durchzufuihren, was sehr hilfreich ist.

Ein Beispiel für ein Suchergebnis zeigt die folgende Abbildung 8.

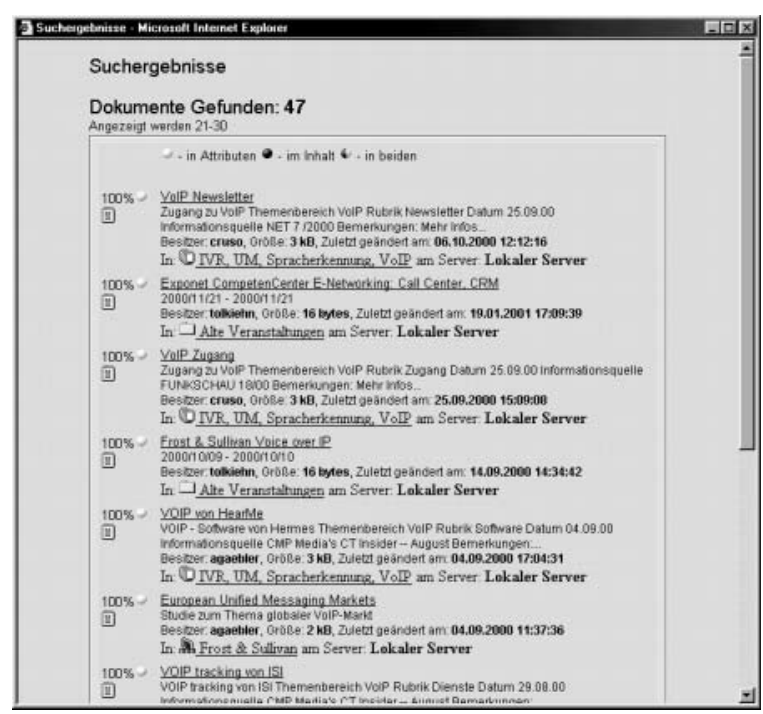

Abb. 8: Screenshot Darstellung der Suchergebnisse. Gesucht wurde nach der Zeichenkette „VoIP“
Das System unterscheidet bei der Suche zwischen dem Vorkommen des Suchbegriffs im Informationsinhalt (z. B. innerhalb einer pdf-Datei) und in einem Attribut (z. B. dem von uns definierten Attribut „Veranstaltungsdatum“ oder dem Standardattribut „Description“). Die Begrenzung der Suche auf den aktuellen Scope ist leider nicht möglich.

- Als zusätzliche Option kann die Suchabfrage auch gespeichert und zeitgesteuert ausgeführt werden. Hierbei kann das Suchergebnis auch in Form einer EMail zugeschickt werden (individueller E-Mail-Newsletter). Man kann sich damit z. B. wöchentlich alles zuschicken lassen, was den Textstring VoIP enthält und innerhalb der letzten sieben Tage dem System hinzugefuigt wurde. Die Suchfunktion zeigte allerdings gelegentlich unerklärliche Ausfallerscheinungen (Objekte wurden nicht gefunden, obwohl sie eindeutig und abrufbar vorhanden waren).

- Benutzer-Anmeldung: Die Benutzeranmeldung ist für den Zugriff auf besondere Bereiche und Funktionen erforderlich, insbesondere für die Einstellung und Änderung von Informationen im System. Den Benutzern können im Einzelnen und gruppenweise in vielfältiger Weise differenzierte Rechte an den Objekten des Systems zugewiesen werden.

- Registrierung für neue Benutzer: Neue Benutzer erhalten ein Formular mit einer Reihe von teilweise optionalen Angaben zur Person und können sich selbst - im Rahmen der Eindeutigkeit - ein User-ID und ein Passwort für ihren späteren Zugang wählen. Die Implementierung dieser Funktion hatte zunächst erhebliche technische Schwierigkeiten bereitet, läuft aber seit Anfang 2000 sehr zufriedenstellend und zuverlässig.

- Explorer: Ein zusätzliches HIS-eigenes Navigationswerkzeug, dass die gesamte Verzeichnisstruktur der Datenbank anzeigt.

- Toolbar: Ein zusätzliches HIS-eigenes sehr mächtiges, aber schlecht dokumentiertes Administrationswerkzeug. Nur für geübte Benutzer geeignet.

- Befragung: Eine in zwei Versionen selbst implementierte Funktion zur einfachen Bewertung der Inhalte durch die Benutzer.

- Add new: Eine kontextabhängige Funktion zum Hinzufuigen neuer Inhalte. Sie erscheint nur, wo diese Möglichkeit implementiert ist und wenn der Benutzer die erforderlichen Rechte hat.

- Löschen und editieren von Objekten: Weitere Funktionen, die als kontextabhängige Buttons implementiert sind, jedoch nur innerhalb der geöffneten Objekte zur Verfuigung stehen, soweit der Benutzer die entsprechenden Rechte hat.

Eine ursprünglich ebenfalls geplante „instant messaging"-Funktion für registrierte Benutzer erwies sich als zu schwierig zu realisieren. Auch die im Jahr 2000 von verschiedenen großen Anbietern in Umlauf gebrachten Instant Messaging-Dienste halfen dabei nicht weiter. Von ihr blieb lediglich die Möglichkeit festzustellen, welche Benutzer gleichzeitig online sind, die aber in der ,Explorer"-Oberfläche versteckt und nur den Systemadministratoren zugänglich ist. 
Darüber hinaus gibt es eine Reihe von Informationen und Funktionen, die nur den Systemadministratoren zur Verfuigung stehen. Bis auf die Funktion „Liste der registrierten Benutzer" wurden sie nicht speziell implementiert. Es wurden hierfuir die HIS-Standardfunktionen im Toolbar verwendet.

\subsection{Benutzer und Statistik}

Es erwies sich anfangs als technisch schwierig, die vorgesehene und für den Erfolg des Systems zwingend erforderliche selbständige Registrierung von Benutzern mit eigener Wahl von User-ID und Passwort zu realisieren. Das Problem bestand in der korrekten Übertragung der verschliusselten Passworte. Nach umfangreicher, aber wenig erfolgreicher Inanspruchnahme des Services von Hyperwave und diversen eigenen Experimenten gelang es schließlich, die heute noch in Betrieb befindliche, sehr zuverlässige Benutzerregistrierung zu realisieren. Ein automatischer Abgleich mit anderen Verzeichnissen innerhalb der TFH Wildau ist jedoch nicht möglich. Bei der Benutzeranmeldung werden dem Benutzer verschiedene Angaben ermöglicht. Einige davon sind obligatorisch, andere optional. Von den optionalen Angaben sind einige innerhalb des Systems durchsuchbar.

Der Benutzer wird durch den Aufbau der Registrierung dazu angehalten, seine Angaben wahrheitsgemäß zu machen. Anonyme Anmeldungen oder offensichtliche Phantasienamen sind technisch möglich, werden aber nicht akzeptiert. Dies wird in regelmäßigen Abständen von den Systemadministratoren geprüft. Wir konnten bisher erfreulicherweise nur in ganz geringem Umfang Umgehungen dieser Richtlinie und noch keinerlei Vandalismus in der Community feststellen.

Zur Information uiber das Benutzerverhalten wurde zunächst mit dem Analysewerkzeug Webtrends auf den vom HIS generierten Logfiles gearbeitet. Es lieferte eine ganze Reihe von wertvollen Informationen, vor allem über das Mengengerüst der Zugriffe. Diese Informationen werden automatisch auf einem zweiten Web-Server generiert und abgelegt.

Zu Ende der Projektlaufzeit betrug das monatlich heruntergeladene Datenvolumen ca. 1,5 Gbyte und die Anzahl der Seitenimpressionen lag knapp unter 20.000 im Monat. Die durchschnittliche Länge einer Benutzersitzung lag in 2001 bei 24 Minuten, was erfreulich ist und auf eine intensive Befassung der User mit der Community schließen lässt.

Aufgrund der doch nur eingeschränkten Möglichkeiten der uns zur Verfuigung stehenden kommerziellen Statistikanalysetools der unteren Preiskategorie wurde zusätzlich eine Reihe von speziellen eigenen Auswertungen und Funktionen spezifiziert und von Studenten bzw. vom Projektteam ausgefuihrt. Diese Untersuchungen ergaben wichtige zusätzliche Hinweise auf das Benutzerverhalten.

Zu Beginn hatten wir die meisten Inhalte nur fuir Mitglieder zugänglich gemacht, jedoch mit sichtbaren Links und für Suchmaschinen durchsuchbar. Aufgrund der Auswertungen des Benutzerverhaltens (hohe Abbruchzahlen bei der Aufforderung zur Registrierung als neues Mitglied) sind wir jedoch Mitte 2000 zu dem Schluss gekommen, die Mehrzahl der Inhalte auch für anonyme Benutzer zu öffnen, um zunächst den Bekanntheitsgrad des Systems zu steigern und die Mitgliedschaft hauptsächlich für die interaktiven Funktionen und die aktive Mitwirkung (als Autor bestimmter Inhalte) vorzusehen.

Dies wird auch genutzt. Der Umfang der Nutzung dieser Möglichkeit ist für die einzelnen Bereiche sehr unterschiedlich, entspricht jedoch insgesamt bei weitem nicht den ursprünglichen Erwartungen. Hierfür gibt es nach unseren Erkenntnissen zwei wesentliche Gründe. Ein Grund ist mit Sicherheit die bisher noch zu fehleranfällige und immer noch zu zeitraubende Arbeitsweise der Informationseingabe. Man benötigt für die Eingabe z. B. einer Veranstaltungsinformation insgesamt ca. 5 Minuten fuir den Aufruf, das Ausfuillen und das Abschicken des Formulars. Bei durchschnittlich jeder dritten Eingabe kommt es jedoch bisher noch zu fuir den Benutzer völlig unerklärlichen Fehlern, z. B. durch nicht abgefangene Formatfehler bei der Dateneingabe. Solche Fehler führen zu einem Verlust der gesamten Eingabe. Die Erstellung einer Kopie einer erfolgreich eingegebenen Information z.B. fuir eine zweite Veranstaltung gleichen Inhalts zu einem anderen Termin und an einem anderen Ort erfordert bisher zusätzliche Kenntnisse, eine spezielle Anwendung auf dem PC des Benutzers (was heutzutage völlig unakzeptabel ist) und ebenfalls ca. zwei Minuten.

Damit indirekt zusammen hängt das zweite und grundsätzlichere Problem für die Benutzerakzeptanz, das heute alle portalartigen Anwendungen kennen: Auch professionelle Benutzer, die regelmäßig ihre eigenen Informationssysteme pflegen, finden es nicht besonders angenehm, die gleiche Information noch in unterschiedliche Formulare womöglich mehrerer fremder Systeme einzugeben, selbst dann nicht, wenn dies einfach ist und problemlos funktioniert. Hier muss eine Kosten-NutzenRelation nachweisbar sein um diese Arbeit zu motivieren, und das ist relativ schwierig zu erreichen.

\subsection{Fortschritte an anderer Stelle}

Eine Reihe von Websites mit verwandten Themen wurden im Laufe des Projekts und auch noch danach in die Informationssammlung aufgenommen (Siehe Ziffer (1), Produkte und Info / CT-Seiten im Web). Einige davon befassen sich auch mit der herstellerunabhängigen Bereitstellung von Produktinformationen, andere sind eher kommunikations- oder veranstaltungsorientiert, weitere sind eher Online-Magazine oder Newsletter, einige haben auch Community-Merkmale, sind jedoch eher Webseiten von herkömmlichen Vereinen (z. B. Call-Center-Forum, Help-Desk-Forum).

Generell scheinen die technischen Probleme, vor allem Systemaufbau, Betriebssicherheit, einfache Handhabung und übersichtliche und ansprechende Darstellung der 
Inhalte heute weitgehend (natuirlich mehr oder auch weniger gelungen) gelöst zu sein. Hauptproblem aller vergleichbaren Systeme ist offenbar heute die Finanzierung der laufenden Kosten, vor allem der für die erheblichen redaktionellen Aufwendungen zur Pflege der Inhalte.

\subsection{Publikation der Ergebnisse}

Das Vorhaben selbst besteht ja in einer Veröffentlichung der angefertigten Sammlung von Informationen und der dazugehörigen Funktionen im Internet. Auf die Effizienz dieser Veröffentlichung wurde naturgemäß in erster Linie Wert gelegt. Der Erfolg dieser Veröffentlichung ist auch durch den Benutzerzuspruch (siehe dazu o. g. Ziffer 3.3) und das gute Ranking in den Internet-Suchmaschinen belegt.

Neben der bereits während der Projektlaufzeit und danach erfolgten öffentlichen Präsentation des Vorhabens durch Vorträge und Exponate auf Fachmessen und Konferenzen (Details dazu in der o. g. Ziffer 2.3), die auch eine gewisse Presseabdeckung erfuhr, ist vorgesehen, den Abschlussbericht des Projekts in den diesjährigen Wissenschaftlichen Beiträgen der TFH Wildau zu veröffentlichen.

\subsection{Kooperationspartner, Drittmittel und Verbesserung der Drittmittelfähigkeit der TFH Wildau durch das Vorhaben}

In dem Vorhaben wurde mit einer ganzen Reihe von Unternehmen aus der CT-Branche zusammengearbeitet, die als Mitglieder der Community ein eigenes Verzeichnis für die Darstellung ihrer Produkte erhalten haben. Diese Unternehmen sind mit dem bekannten Nord-SüdGefälle über ganz Deutschland verteilt. Darüber hinaus wurde mit der dc Congress Gesellschaft in München in besonderer Weise kooperiert, um die Darstellung der Community auf den Fachmessen Exponet 1999 (in Düsseldorf) und Exponet 2000 (in Köln) zu ermöglichen.

In das Projekt sind außer der Förderung durch das BMBF und die Eigenleistungen der TFH Wildau keine Barmittel von dritter Seite eingeflossen. Jedoch wurden von Anfang an in erheblichem Umfang Sachleistungen und Dienstleistungen fuir das Vorhaben zur Verfuigung gestellt. Hierbei sind besonders zu nennen die Software-Unternehmen Hyperwave AG, München, mit der unentgeltlich zur Verfügung gestellten Softwarelizenz für das Content-Management-System und die PRS-GmbH, Karlsruhe, (heute nicht mehr im Geschäft) mit der Spende eines kompletten Serversystems, das Institut für Telekommunikation im Technologie- und Weiterbildungszentrum e.V. an der TFH Wildau, das ein PC-System und einiges an Kleinmaterial zur Verfuigung gestellt hat und die dc Congress Gesellschaft, die in 1999 und 2000 Ausstellungsstände fuir das Vorhaben unentgeltlich zur Verfügung gestellt hat. Der Anschaffungswert dieser zugewendeten Sach- und Dienstleistungen liegt insgesamt bei etwa 50 TDM.

In den mit einer Reihe Unternehmen aussichtsreich gefuihrten Verhandlungen über eine direkte Förderung der
Community mit Barmitteln zur weiteren Entwicklung und Pflege des Systems nach Ende des Förderzeitraums erlebten wir in der zweiten Hälfte 2000 und in 2001 durch eine Reihe von Insolvenzen der betreffenden Unternehmen einen Ruickschlag. Das wirtschaftliche Konzept und auch die Fortfuihrung des Vorhabens sind dadurch jedoch nicht grundsätzlich in Frage gestellt.

Unabhängig von dieser noch nicht ganz erwartungsgemäßen wirtschaftlichen Situation hat sich das Projekt und die damit verbundene Publicity für die Drittmittelfähigkeit der TFH und des Technologie- und Weiterbildungszentrums bereits sehr positiv ausgewirkt. Wir haben in Zusammenhang mit dem Vorhaben bereits eine ganze Reihe von Anfragen für Projektkooperationen von Unternehmen erhalten, aus denen auch bereits drei konkrete kleinere Aufträge fuir Web-basierte Informationssysteme und einer fuir ein CD-ROM-basiertes System resultierten, die wir mithilfe von Studenten und Mitarbeitern sowie einem KMU aus der Region realisieren konnten.

Auch mehrere Angebote fuir Industriekooperationen im Rahmen der Lehre mit Sponsoring von Hardware, Software und Dienstleistungen aus dem Bereich ComputerTelephony für unsere Labore waren bisher das Ergebnis des Projekts. Eines davon wurde bereits in Angriff genommen. Die Arbeit an solchen Lösungen können wir den Studenten aus Eigenmitteln der TFH aufgrund der hohen Anschaffungskosten in der Regel nicht ermöglichen. Sie gibt den Studenten aber aktuellen Einblick in komplexe Systeme und ist dadurch fuir die Ausbildung in der Wirtschaftsinformatik und Telematik sehr wertvoll.

\section{Danksagung}

Dem BMBF danke ich für die Förderung des Projekts. Den Mitarbeiterinnen und Mitarbeitern des Projektträgers AiF sowie dem Leiter unseres Haushalts, Herrn Thomas Lehne, und seinen Mitarbeiterinnen möchte ich für die unkomplizierte und verständnisvolle Unterstuitzung bei der verwaltungstechnischen Abwicklung des Projekts danken. Ebenso danke ich besonders unserer Laboringenieurin Frau Susanne Seipold und den Mitarbeitern Alexej Okonetshnikov, Nadine Röhtz, Andreas Gäbler, Elena Moukhina, Ivan Issaev und auch den vielen Studenten, die in dem Projekt involviert waren für Ihre engagierte Arbeit und unseren Sponsoren für ihre großzuigige Unterstuitzung sowie vor allem den Mitgliedern der Community für ihr Interesse, ohne das das Projekt nicht in der vorliegenden Form möglich gewesen wäre.
Autor
Prof. Dr. Günter-Ulrich Tolkiehn
Technische Fachhochschule Wildau
Fachbereich Betriebswirtschaft/Wirtschaftsinformatik Tel. (0 33 75) 508-239
E-Mail: tolkiehn@tfh-wildau.de 